\title{
Appréciation du bois de chêne (Quercus robur L, Quercus petraea Liebl) par les consommateurs et les professionnels français du bois
}

\author{
R Marchal ${ }^{1}, \mathrm{~F}$ Mothe ${ }^{2 *}$ \\ 1 ENSAM - Arbre, place du 11-Août, 71250 Cluny; \\ 2 INRA - Recherches forestières - qualité des bois, 54280 Champenoux, France
}

(Reçu le 5 avril 1992 ; accepté le 3 mars 1993)

\begin{abstract}
Résumé - La ressource de chêne évolue. Les scieurs français produisent davantage de débits de "qualités intermédiaires" comportant des singularités. Ceux-ci ne trouvent pas de débouchés suffisants faute d'une connaissance fine des goûts du public en matière de bois de chêne. Pour combler ce manque, une enquête faisant appel à la comparaison par paires de 100 frises a été menée auprès de 566 personnes - dont 94 professionnels du bois. Des analyses factorielles des correspondances et des observations directes de paires ont permis de constater que les réponses ne sont pas uniformes: tous les goûts sont fortement représentés ; les critères participant aux choix formulés par les personnes seraient, dans un ordre d'importance décroissant, la nodosité, l'orientation de coupe, la clarté, l'homogénéité de teinte, la largeur des cernes ; 2 populations de consommateurs existent : ceux rejetant tout nœud (professionnels du bois, bricoleurs...) et ceux préférant le chêne assez faiblement noueux à des bois sans nœud (population plus féminine) ; plus la personne est âgée, plus ses choix suivent les critères traditionnels de qualité ; les catégories socio-professionnelles ne se différencient pas significativement par leur comportement. Les seconds transformateurs sont donc assurés de satisfaire une large clientèle en intégrant des choix QF1bis, voire QF2 en parement.
\end{abstract}

chêne / frises / aspect / enquête / consommateur

Summary - Appreciation of oak wood (Quercus robur L, Quercus petraea Liebl) for the French consumer and wood professionals. French sawers always produce more cuttings of intermediate quality. These products are not marketable because of the lack of a fine knowledge of people's tastes regarding oakwood. A total of 566 persons, including 94 professionals, were thus asked about their visual appreciation of a hundred strips of wood. Multiple correspondence analyses and direct observation of pairs have allowed us to establish the following. There was a great variety in the answers; all kinds of tastes coexist. The criteria affecting people's choice are, in

\footnotetext{
* Correspondance et tirés à part.
} 
decreasing order: nodosity; cut orientation; tint; and annual ring width. There are 2 groups of consumers, one rejecting all marks of knots (professionals and handymen), the other is more feminine, preferring a slightly knotty wood rather than pieces free of knots. Elderly people had more traditional choices. It was not possible to establish a specific behaviour according to classical socio-professional categories. There is thus a large number of people quite capable of preferring small knots in the face of solid oak.

\section{oak / strip / visual appearance / survey / consumer}

\section{INTRODUCTION}

La ressource sur pied du bois de chêne évoluant, les scieurs français produisent toujours plus de débits de «qualités intermédiaires» comportant, entre autres singularités, des nœuds de taille et fréquence variables.

Ces produits ne trouvent pas de débouchés suffisants, en partie faute d'une connaissance fine des goûts du public en matière d'esthétique du bois de chêne. Nous entendons souvent dire que le consommateur n'apprécie le chêne en emploi apparent qu'à condition qu'il soit exempt de tout défaut: teinte homogène, absence de nœud, droit de fil, etc.

S'il est certain que tous ces critères interviennent dans le choix des personnes, nous ignorons leur importance respective.

Nous nous proposons de vérifier les affirmations courantes qui pénalisent fortement les premiers transformateurs - inspirateurs de cette étude - ainsi que les tenants d'une sylviculture dynamique du chêne, et d'établir une hiérarchie dans les divers critères de choix, par un travail d'enquête sur échantillons de bois, essentiellement menés auprès de consommateurs " non avertis" de bois de chêne.

Un complément d'enquête dans les milieux professionnels du bois a été effectué suivant un protocole tout à fait identique, afin d'identifier les éventuelles divergeances de vue entre les 2 "populations".

Notre approche du problème s'inspire en partie de celle de Mazet (1988) cherchant à connaître l'influence de la couleur des placages d'ébénisterie de chênes sur le choix des professionnels producteurs ou utilisateurs de ces placages.

Elle en diffère du fait de la dimension d'«analyse du marché» que nous mettons ici en avant dans le cadre de recherches de débouchés pour des bois de chêne de qualité secondaire.

\section{MATÉRIEL ET MÉTHODE}

Le protocole d'enquête choisi est du type de celui décrit par Brun-Chaize (1978) et par Mazet (1989 ; et Mazet et Janin, 1990) : la comparaison d'échantillons par paires et le traitement des données par analyses factorielles des correspondances.

L'avantage de la comparaison par paires est de permettre la présentation d'un grand nombre d'objets aux personnes ciblées sans pour autant trop les fatiguer. Chaque comparaison ne porte en effet que sur deux éprouvettes : il n'est pas nécessaire de se référer à toutes les autres.

\section{Le matériel d'enquête : les frises}

Cent débits de chênes médio-européens (Quercus robur L, Quercus petrae Liebl), séchés à environ $12 \%$, ont été sélectionnés aux Scieries réunies du Châlonnais (Givry, Saône-et-Loire) par leur PDG Dominique Juillot, afin d'obtenir une représentation équitable dans les 4 classes définies par la prénorme de l'APECF (1989):

$-25 \%$ en QF1, sciages de droit fil exempts de toute singularité sur 3 faces ;

$-25 \%$ en QF1 bis où quelques petits nœuds, voire de rares nœuds de taille moyenne, sont tolérés ; 
- $25 \%$ en QF2 renfermant des petits nœuds en nombre non limité ou de gros nœuds, rares, sains et adhérents ;

$-25 \%$ en QF3 tolérant de plus gros noeuds sains et adhérents.

Pour ces 4 catégories, l'aubier, la moelle et les altérations sont exclus.

Les débits ont ensuite été calibrés en frises de $100 \mathrm{~mm} \times 850 \mathrm{~mm} \times 20 \mathrm{~mm}$, puis soigneusement poncés. En présentant des échantillons de chêne de taille et finition rigoureusement identiques, nous sommes assurés que les différences de jugement porteront uniquement sur l'aspect du bois.

Un tirage aléatoire a permis de constituer 50 paires de frises numérotées de $1 \mathrm{~A}$ à $50 \mathrm{~B}$, référencées par un marquage laser en creux, afin de pouvoir rafraîchir par ponçage autant que nécessaire la face présentée au public.

\section{Les relevés sur frises : les données objectives}

Dix-huit données ont été mesurées ou calculées sur la face à tester de chaque frise. Une $19^{e}$ donnée, la densité de la frise, a été adjointe à cette liste. La densité du bois étant fonction de l'anatomie, sa mesure pourrait théoriquement intégrer des caractéristiques d'aspect du bois.

Ces 19 données permettront d'expliquer les choix subjectifs formulés par le public.

II s'agit des caractéristiques suivantes:

- APE : le classement suivant la prénorme de l'APECF ;

- OR| : l'orientation dominante du débit (dosse, quartier...);

- LGC : la largeur moyenne des cernes participant au figuré ;

- NBC : le nombre de cerne participant au figuré ;

- NBN : le nombre de nœuds visibles sur la face présentée ;

- \%SN : la proportion de la surface occupée par les nceuds ;

- $\sigma S N$ : l'écart type de la surface des nœuds ;

- CVN : le coefficient de variation de la surface des nouds ;

- SMN : la surface d'un nœud moyen ;

- ISN : l'indice de répartition spatiale des nœuds ;
- L* 7 les trois coordonnées chromatiques

$\left.-a^{*}\right\}$ dans le système de couleur Cielab

$-b^{*} \int$ (Kowaliski, 1990)

$-h^{*}$ : l'angle métrique de teinte ;

$\left.-\sigma L^{*}\right\}$ les écarts types des 3 coordonnées $\left.-\sigma a^{*}\right\}$ chromatiques ;

$-\sigma b^{*}$

- $\sigma h^{*}$ : l'écart type de l'angle métrique de teinte

- DEN : la densité globale à $12 \%$ d'humidité ;

(Voir modèle opératoire, fig 1).

APE est le résultat du tri de Dominique Juillot.

Les résultats concernant ORI, LGC et NBC sont des moyennes entre les observations faites sur les 2 sections transversales de chaque frise. LGC et NBC concernent uniquement les cernes apparents sur la face présentée.

Les surfaces de nœuds ont été mesurées à partir des jeux d'ellipses mis à disposition par le département «Bois et sciages» du CTBA.

L'indice ISN, ainsi que les mesures de couleur, ont été déterminés à l'aide d'une trame divisant la surface des frises en 8 carrés de $100 x$

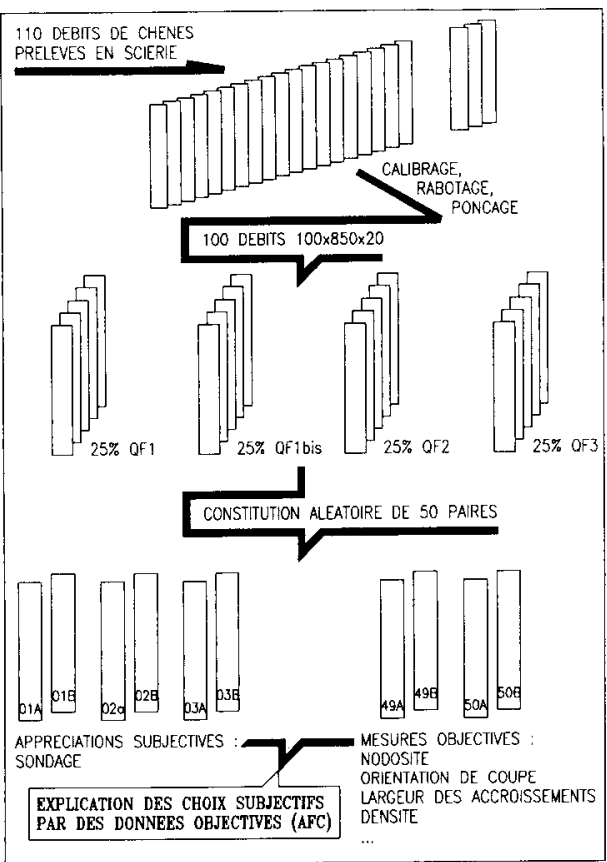

Fig 1. Mode opératoire. 
$100 \mathrm{~mm}^{2}$. ISN est l'écart type du nombre de nœuds comptés dans chaque carré.

Les caractéristiques de couleur ont été mesurées sur un spectrocolorimètre dont le principe et le fonctionnement ont été décrits par Janin (1987). Rappelons que le système Cielab à 3 coordonnées a été établi pour se rapprocher de la vision de l'œil humain. Ainsi :

- L*, clarté métrique, varie de 0 pour le noir à 100 pour le blanc de référence;

- a* est la coordonnée sur l'axe allant du vert (-) au rouge $(+)$;

$-b^{*}$ est la coordonnée sur l'axe allant du bleu $(-)$ au jaune (+) ;

$-h^{*}$, l'angle métrique de teinte, est calculé à partir de $a^{*}$ et $b^{*}$ pour donner des informations quant à la tonalité de la couleur de l'échantillon $: h^{*}=$ $\operatorname{arctg}\left(b^{*} / a^{*}\right)$.

Deux mesures distantes de $2 \mathrm{~cm}$ des bords ont été effectuées en évitant les ncuds à micôté de chaque carré de la trame décrite ci-dessus (16 mesures par frise et par critère). Les critères décrivant la couleur sont des moyennes et écarts types calculés à partir de ces 16 mesures. (Diamètre de la fenêtre d'exploration : $9 \mathrm{~mm}$. Illuminant choisi : D65.)

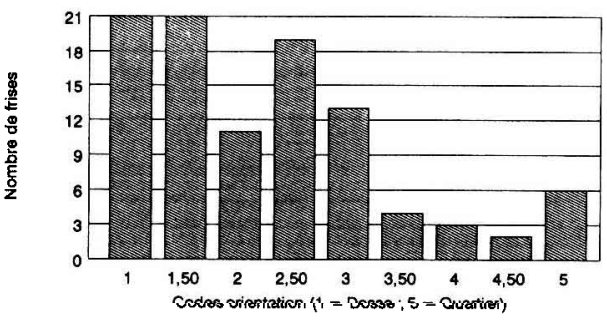

Fig 2. Variabilité de l'échantillonnage pour l'orientation de coupe (ORI).

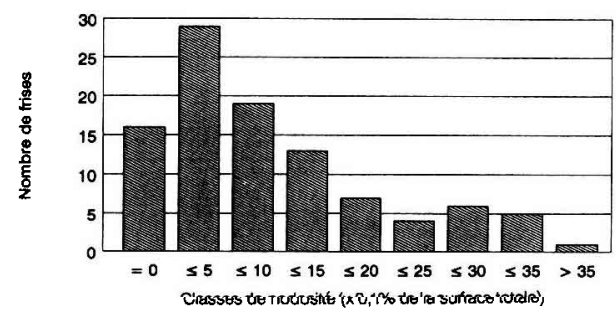

Fig 4. Variabilité de l'échantillonnage pour la proportion de la surface occupée par les nœuds (\%SN).
Les écarts types et les coefficients de variation des 3 coordonnées chromatiques et de l'angle métrique de teinte ont été calculés. Seuls les écarts types ont été conservés car ils se sont révélés plus discriminants.

Les calculs d'écarts de couleur, de chromaticité, de teinte entre les 2 échantillons d'une même paire $(\Delta \mathrm{E}, \Delta \mathrm{C}, \Delta \mathrm{H}) \mathrm{n}^{\prime}$ ont pas été conservés ici car les valeurs, par définition positives, ne permettent pas de construire un classement à l'intérieur de chaque paire et donc ne participent pas suffisamment à l'explication des choix subjectifs des personnes.

La variabilité de l'échantillonnage est décrite dans le tableau I, complété par les figures 2 à 5 .

\section{La population soumise à l'enquête}

Elle se compose de 472 consommateurs potentiels contactés pour l'essentiel en Bourgogne et en Lorraine, 2 des plus grandes régions françaises productrices de chêne.

Nous avons complété cet échantillonnage par 94 professionnels connaissant le travail du chêne, et rencontrés lors du salon Expobois 92 à Paris,

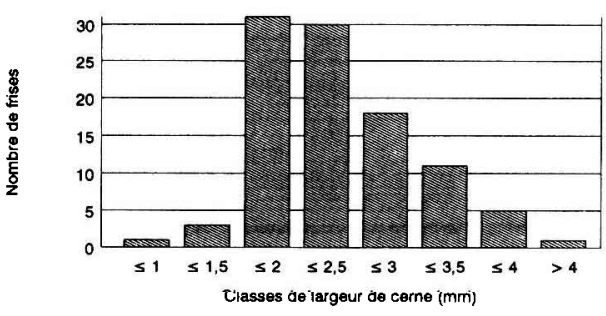

Fig 3. Variabilité de l'échantillonnage pour la largeur de cerne (LGC).

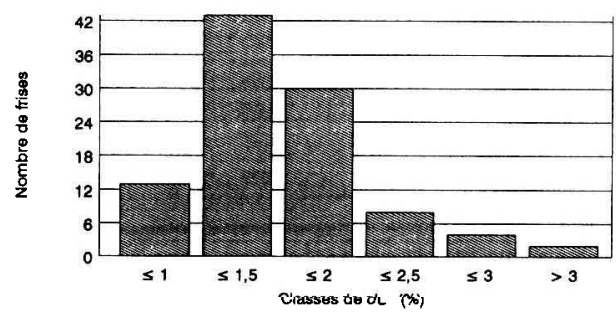

Fig 5. Variabilité de l'échantillonnage pour l'écart type de la clarté $\left(\sigma L^{*}\right)$. 
Tableau I. Valeurs minimales, maximales, moyennes et écarts types des variables objectives caractérisant les 100 frises.

\begin{tabular}{|c|c|c|c|c|c|}
\hline Critères & Unités ou codes & Minimum & Maximum & Moyenne & Écart type \\
\hline APE & $\begin{array}{c}\text { QF1 }=1 \\
\text { QF1bis }=1,5 \\
\text { QF2 }=2 \\
\text { QF3 }=3\end{array}$ & 1 & 3 & 1,88 & 0,75 \\
\hline DEN & su & 0,576 & 0,853 & 0,704 & 0,053 \\
\hline ORI & $\begin{array}{c}\text { Dosse }=1 \\
\text { Intermédiaire }=2 \\
\text { Faux quartier }=3 \\
\text { Intermédiaire }=4 \\
\text { Quartier }=5\end{array}$ & 1 & 5 & 2,26 & 1,12 \\
\hline $\begin{array}{l}\text { LGC } \\
\text { NBC }\end{array}$ & $\begin{array}{l}\mathrm{mm} \\
\mathrm{SU}\end{array}$ & $\begin{array}{c}1,00 \\
4,5\end{array}$ & $\begin{array}{l}4,13 \\
50,5\end{array}$ & $\begin{array}{l}2,34 \\
21,5\end{array}$ & $\begin{array}{l}0,64 \\
11,6\end{array}$ \\
\hline $\begin{array}{l}\text { NBN } \\
\% S N \\
\sigma S N \\
\text { CVN } \\
\text { SMN } \\
\text { ISN }\end{array}$ & $\begin{array}{c}\mathrm{SU} \\
\% \\
\mathrm{~mm}^{2} \\
\% \\
\mathrm{~mm}^{2} \\
\mathrm{SU}\end{array}$ & $\begin{array}{l}0 \\
0 \\
0 \\
0 \\
0 \\
0\end{array}$ & $\begin{array}{c}40 \\
58 \\
588 \\
334 \\
492 \\
3,8\end{array}$ & $\begin{array}{c}10 \\
10 \\
106 \\
92 \\
86 \\
1,1\end{array}$ & $\begin{array}{c}9,0 \\
10,5 \\
138,4 \\
77,8 \\
100,0 \\
0,8\end{array}$ \\
\hline $\begin{array}{l}\mathrm{L}^{*} \\
\mathrm{a}^{*} \\
\mathrm{~b}^{*} \\
\mathrm{~h}^{*} \\
\sigma \mathrm{L}^{*} \\
\sigma \mathrm{a}^{*} \\
\sigma \mathrm{b}^{*} \\
\sigma \mathrm{h}^{*}\end{array}$ & $\begin{array}{c}\% \\
\text { SU } \\
\text { SU } \\
d^{\circ} \\
\% \\
\text { SU } \\
\text { SU } \\
d^{\circ}\end{array}$ & $\begin{array}{c}64,1 \\
9,3 \\
21,0 \\
62,6 \\
0,6 \\
0,2 \\
0,3 \\
0,2\end{array}$ & $\begin{array}{c}71,5 \\
11,6 \\
24,0 \\
66,4 \\
3,1 \\
0,7 \\
1,2 \\
1,0\end{array}$ & $\begin{array}{c}68,1 \\
10,6 \\
22,8 \\
65,1 \\
1,5 \\
0,4 \\
0,6 \\
0,5\end{array}$ & $\begin{array}{l}1,6 \\
0,4 \\
0,6 \\
0,7 \\
0,5 \\
0,1 \\
0,2 \\
0,1\end{array}$ \\
\hline
\end{tabular}

ou à l'occasion de diverses autres manifestations régionales.

La typologie de cette population de 566 personnes - comparée aux données nationales fournies par I'INSEE pour la France métropolitaine (recensement de 1990) - est résumée sur les figures 6 à 8.

If en ressort les observations suivantes:

- la population sondée représente exactement un $100000^{\mathrm{e}}$ de la population française ;

- la répartition hommes/femmes est quasiment identique à celle donnée par l'INSEE : $48,1 \%$ et $51,9 \%$ contre $48,7 \%$ et $51,3 \%$;

- les classes d'âge les plus extrêmes sont sousreprésentées ; concernant les moins de 20 ans, cela est la conséquence d'un choix délibéré, le test n'ayant pas été conçu pour une population enfantine ;

- l'âge moyen est légèrement supérieur à la moyenne nationale : 39 ans contre 36 ;

- la classe "cadres supérieurs et professions libérales" est sur-représentée au détriment de la classe "ouvriers".

\section{Le questionnaire : les données subjectives}

Chaque individu a été au préalable classé parmi les 5 catégories socio-professionnelles citées en 
figure 7, voire dans les catégories "étudiants" et "sans profession". Ces renseignements ont été complétés par 4 informations complémentaires concernant l'appartenance ou non à un métier du bois, l'âge, le sexe et la propension de la personne à travailler le bois dans ses loisirs.

Les objectifs visés par ce sondage ont été ensuite présentés, d'une manière succinte, afin de motiver la personne interrogée sans pour autant influencer ses choix.

Enfin, chaque paire a été soumise au jugement subjectif de l'individu sondé, qui a dû exprimer sa préférence en respectant les quelques règles suivantes :
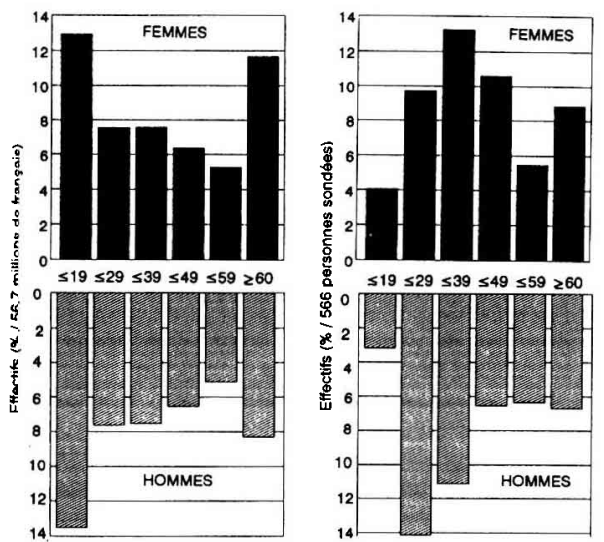

Fig 6. Pyramides des âges pour la population testée et la population métropolitaine française.
- chaque paire a été présentée de manière isolée des autres de manière à éviter toute comparaison avec les paires antérieures ou postérieures ;

- les réponses devaient être strictement individuelles ;

- on ne demandait le jugement de l'individu que par rapport à une seule face des frises; par conséquent, les frises ne pouvaient être retournées pour mieux arrêter son choix ;

- la réponse devait être donnée rapidement, de manière à ce que la personne sondée ne soit pas amenée à analyser le pourquoi de sa réponse; la durée moyenne d'un passage sur les 50 paires avoisinait $10 \mathrm{~min}$;

- seule une des 3 réponses suivantes était admise : la préférence pour la frise $A$; la préférence pour la frise $B$; pas de préférence, dans le sens où aucune différence n'est vue. Cette dernière réponse ne doit être formulée qu'exceptionnellement afin de nous permettre d'identifier des critères fins de choix ;

- la présentation des paires se fait toujours dans le même ordre à une permutation circulaire près, nécessaire pour "noyer" un éventuel biais dû à la lassitude du sujet.

Toutes les appréciations et remarques exprimées au cours d'une session et pouvant a posteriori aider à l'analyse des résultats sont consignées par l'enquêteur.

\section{RÉSULTATS}

Deux types d'analyses complémentaires ont permis de dégager quelques tendances
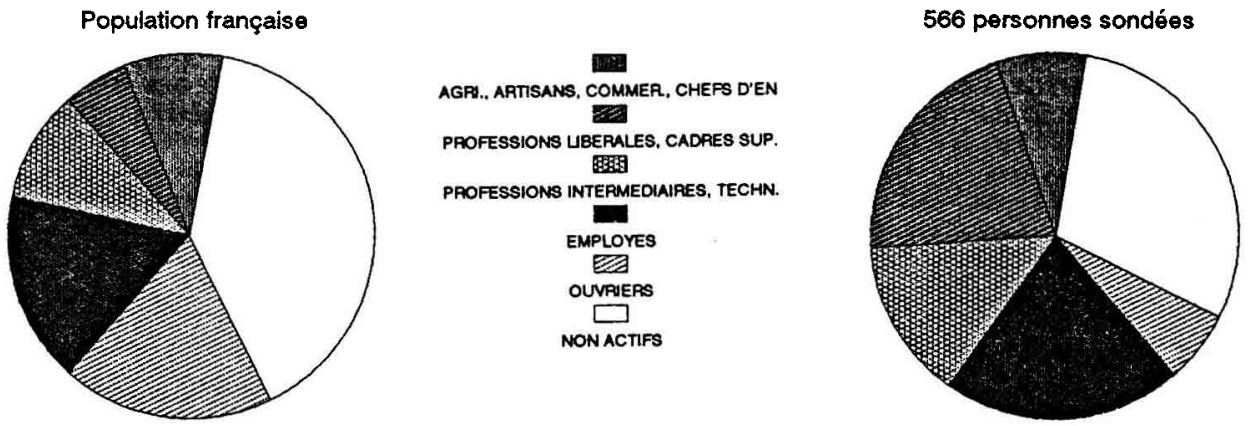

Fig 7. Répartition en 5 catégories socio-professionnelles de la population testée et de la population métropolitaine française (les retraités ont été intégrés dans leur catégorie d'origine). 


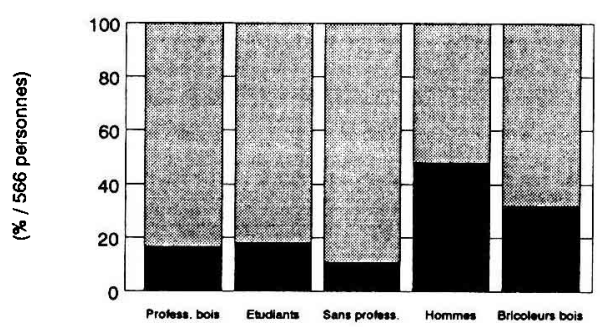

Fig 8. Données complémentaires relatives à la population testée.

nettes. II s'agit d'analyses factorielles des correspondances multiples (AFCM), pour mettre à nu les grands équilibres et les grandes tendances; d'observations directes de paires de frises pour lesquelles les réponses ont été particulièrement typées. Ces observations permettent d'affiner la définition des critères de choix.

\section{Traitement des données}

L'analyse factorielle des correspondances est la technique la mieux appropriée pour le dépouillement d'enquêtes de ce type (Dervin, 1990). Nous avons utilisé la cinquième version du logiciel STAT-ITCF (1991).

La «feuille» d'analyse (fig 9) se présente comme un grand tableau de 566 lignes sur 50 colonnes auxquelles s'ajoutent:

- 7 colonnes supplémentaires décrivant le profil de la personne sondée (catégories socio-professionnelles, âge, sexe...);

- 38 lignes supplémentaires permettant une utilisation simple et originale des données objectives relevées sur les frises (classement APECF, critères de nodosité, couleur...).

En effet, ces derniers paramètres ont été présentés et encodés de la même manière que des réponses d'enquêtes, comme s'il s'agissait de "robots" programmés pour ne donner leur choix qu'en fonction d'un seul critère.
Les conventions suivantes ont alors été adoptées : pour une paire de frises et une caractéristique données, 2 robots sont programmés :

- un «robot positif» dont le choix se porte sur la frise, dont la valeur mesurée est la plus conforme aux idées reçues concernant la qualité du bois de chêne; en général, il s'agit de la valeur la plus faible, à l'exception des critères $L^{*}, a^{*}, b^{*}$ et NBC, pour lesquels le robot choisit la plus élevée (c'est-à-dire respectivement la frise la plus claire, tendant le plus vers le rouge et le jaune, et celle comportant le plus de cernes) ;

- un «robot négatif» qui formule un choix inverse.

Si la différence entre $A$ et $B$ est faible, les 2 robots feront un «non choix».

Le seuil de «non choix» a été fixé à 10\% de la valeur moyenne de chaque paire pour tous les critères objectifs, à l'exception de DEN, $L^{*}, a^{*}, b^{*}$, pous lesquels le seuil a été abaissé à $1 \%$; et $h^{\star}$ pour lequel il a été fixé à $1 \%$, en raison de la faible variabilité de l'échantillonnage pour ces critères.

Nous avons donc ainsi créé 38 robots: 19 robots positifs (notés $X X X^{+}$, où $X X X$ est l'abréviation adoptée précédemment) et 19 robots négatifs $\left(X X X^{-}\right)$.

\section{Les enseignements obtenus par les analyses factorielles des correspondances multiples}

\section{Analyse sur le fichier complet F1 (AFC1)}

La première analyse, notée par la suite AFC1, porte sur la population complète, soit 566 individus.

L'examen des projections des différents nuages (individus, choix, robots et profils sociaux) dans le plan principal 1-2 (fig 10) fait apparaitre 2 groupements des points "choix" étagés sur l'axe 2 : les "non choix» sont tous rassemblés dans la partie infé- 


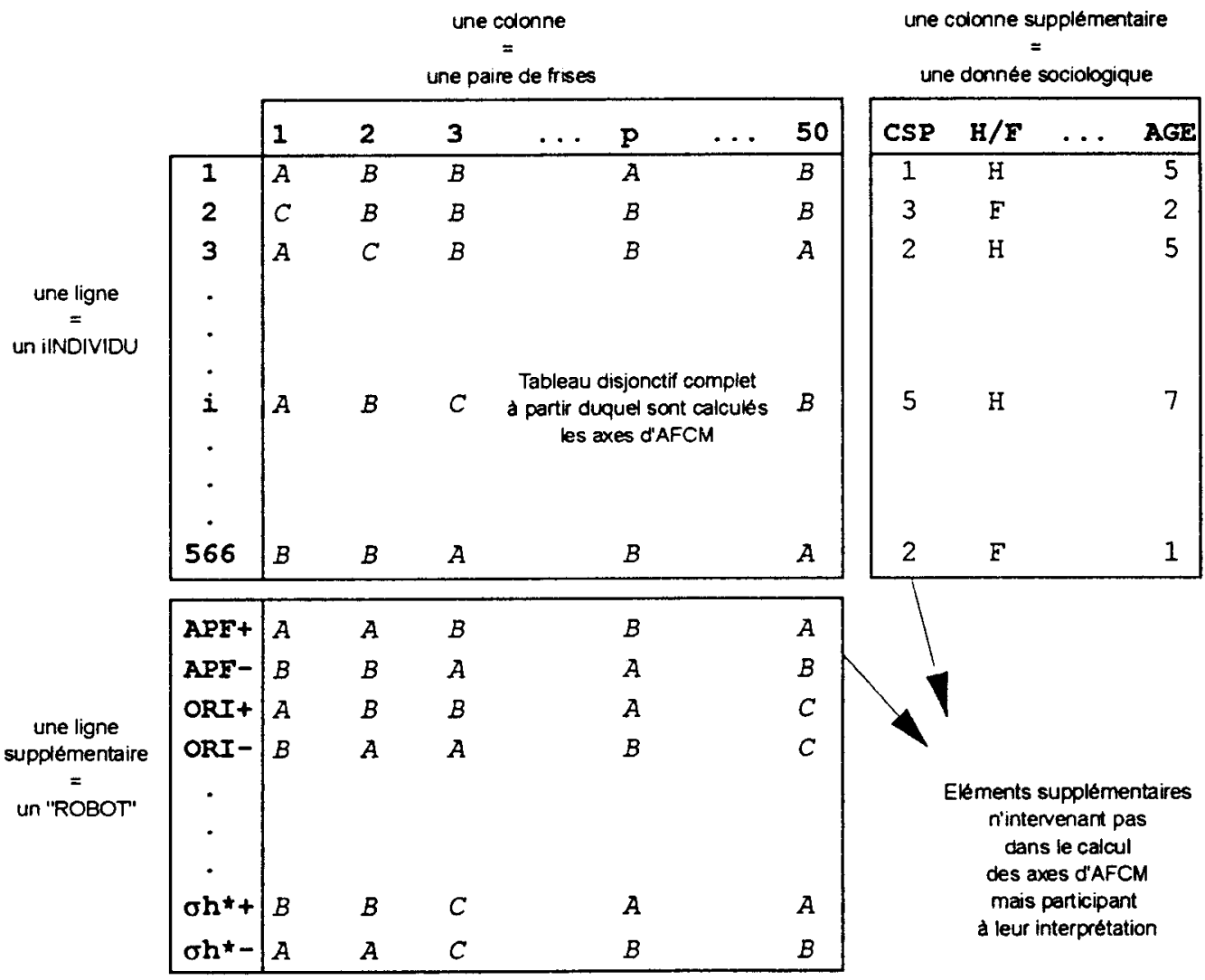

Fig 9. Structure de la feuille d'analyse : relevés d'enquête et mesures objectives présentées sous forme de "robots".

rieure de la projection 11a; de ce fait, les individus se trouvant dispersés dans le même quadrant de la projection $11 \mathrm{~b}$ se caractérisent par leur indécision ; par exemple, les 8 personnes les plus excentrées, numérotées $9,38,28,39,12,97$ et 27 , ont respectivement formulé $25,23,23$, $23,18,17$ et 16 «non choix": ce sont les personnes aux goûts les moins arrêtés parmi la population sondée.

Une exploitation préliminaire des résultats des 120 premières réponses (Marchal et Mothe, 1992) nous a conduits à inciter les personnes suivantes à n'utiliser le «non choix» qu'en ultime recours. En effet, il a été souvent mal interprété par les personnes enquêtées, certaines le comprenant comme une «non préférence» plutôt que comme une absence de différence perceptible entre les 2 frises. D'où un nombre parfois élevé de "non choix" exprimé par une même personne. En d'autres termes, nous avons été conduits à inciter les personnes tentées par de fréquentes «non préférence» à faire preuve d'un plus grand discernement.

Cela explique que les numéros mentionnés ci-dessus soient tous inférieurs à 120. 


\section{A - AFC1: Nuage des Choix}

Axe 2

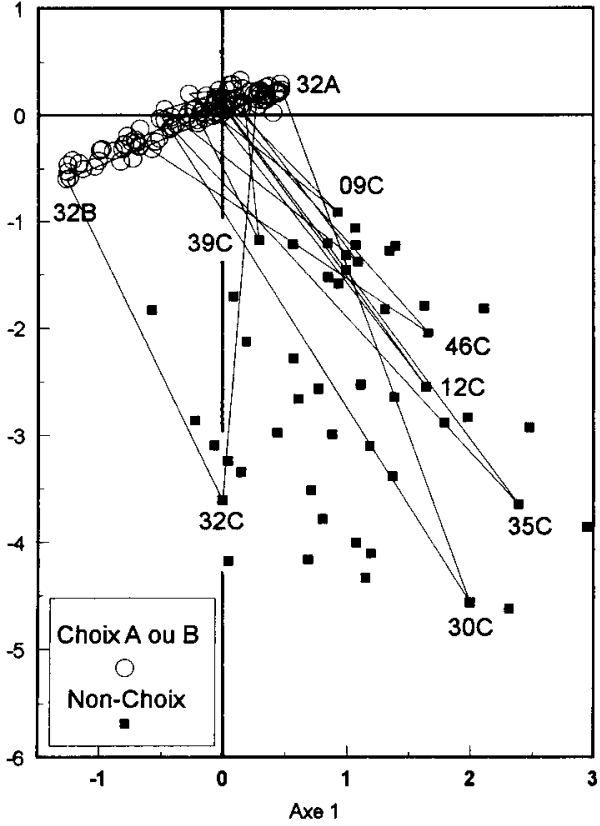

C - AFC1: Nuage des Robots

Axe 2

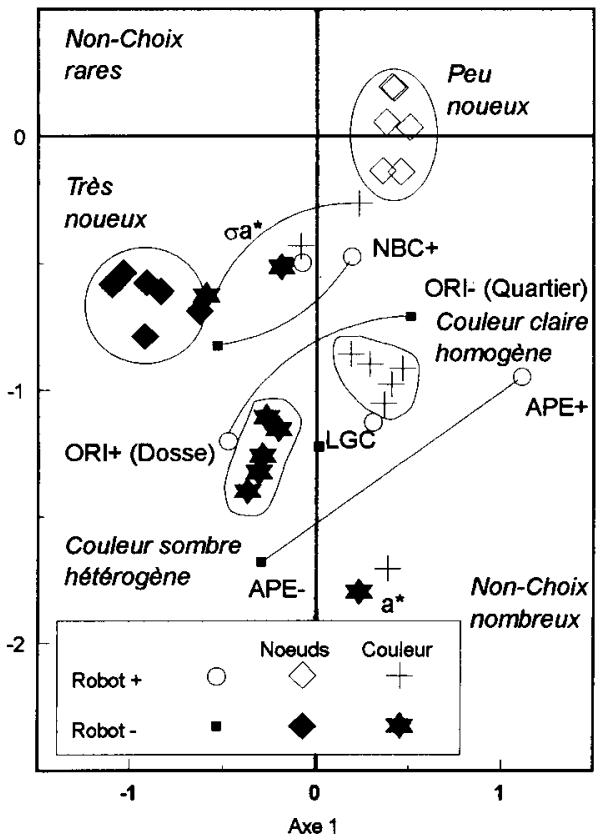

B - AFC1: Nuage des Individus

Axe 2

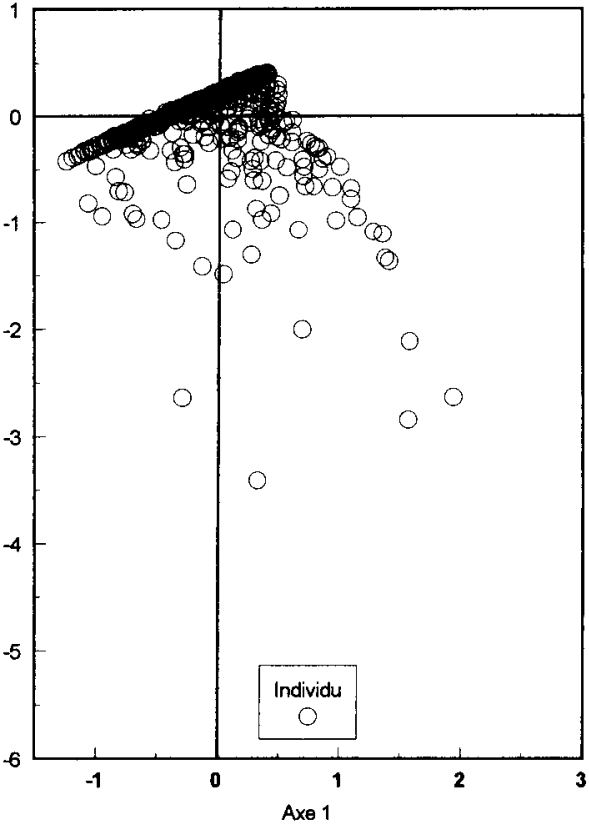

D - AFC1: Nuage des Profils Sociaux Axe 2

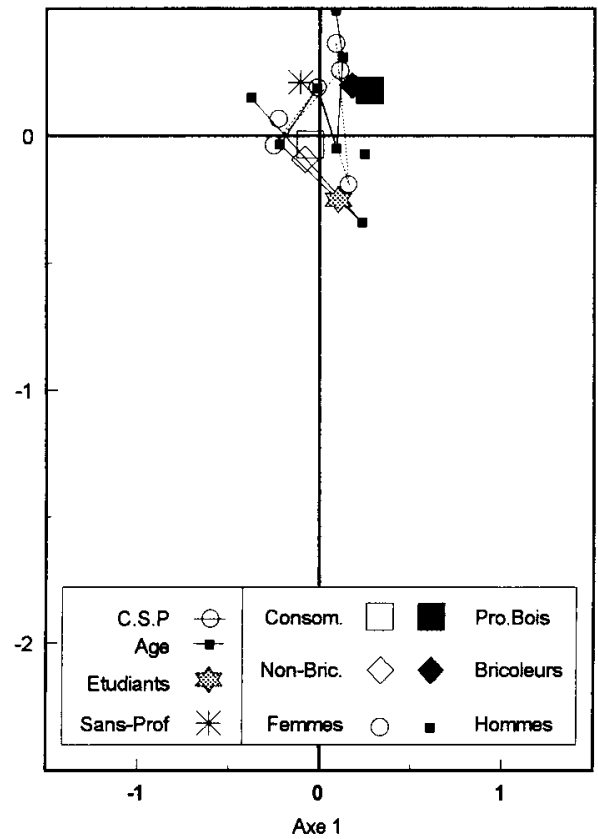

Fig 10. AFC1 : projections dans le plan principal. 
La figure 10 fait également apparaître un allongement du second groupement sur un axe presque confondu à l'axe 1. La figure 10c permet de comprendre la signification de cet axe. II s'agit en fait d'un axe purement qualitatif au sens traditionnel. La partie droite concentre la plupart des robots positifis : $\mathrm{APE}^{+}, \mathrm{LGC}^{+}, \mathrm{NBC}^{+}, \mathrm{NBN}^{+}, \% \mathrm{SN}^{+}$, $\sigma \mathrm{SN}^{+}, \mathrm{CVN}^{+}, \mathrm{SMN}^{+}, \mathrm{ISN}^{+}, \mathrm{L}^{*+}, \mathrm{b}^{{ }^{+}}, \sigma \mathrm{a}^{{ }_{+}}$, $\sigma b^{*+}, \sigma h^{*+}$. La seule exception est $\left.\mathrm{OR}\right|^{-}$, ce qui signifie que l'orientation "plutôt quartier», forcément moins noueuse, est fréquemment associée à la notion de qualité du bois de chêne.

À l'exception de la clarté métrique, les caractéristiques moyennes quantifiant la couleur sont peu discriminantes par rapport à leurs écarts types exprimant l'hétérogénéité de teinte.

L'étagement et le décentrage des robots sur l'axe 2 traduisent simplement l'importance des "non choix" qu'ils ont exprimés: 15 pour APE, 14 pour $\mathrm{a}^{*}$, contre 3 à 7 pour les critères décrivant la nodosité.

Les profils sociaux ne permettent pas d'expliquer la répartition des individus dans le plan principal (projection 11d). II apparaît néanmoins que les professionnels du bois et les bricoleurs se regroupent sur l'axe 1 dans sa partie droite, c'est-à-dire vers le pôle des "choix traditionnels". Une tendance similaire semble séparer les hommes et les femmes.

L'étalement du nuage des individus le long de l'axe 1 traduit la très importante variété des goûts du public. La figure 11 fait ressortir le décalage consommateurs/professionnels du bois : ces derniers ont des goûts plus classiquement admis, exprimés plus unanimement.

L'importance des «non choix», le biais expérimental s'y attachant du fait des ambiguités survenant dans l'interprétation du «non choix», tant par certains sondés que par certains sondeurs, la différence statistique de comportement entre les professionnels $\mathrm{du}$ bois et les consommateurs nous ont conduits à mener d'autres analyses factorielles des correspondances sur des fichiers expurgés des individus trop marqués par la fréquence de leur "non choix", ainsi que sur des fichiers homgènes "professionnels du bois" (analyses AFC\#P) et "consommateurs» (analyses AFC\#C, fig 12).

\section{Analyse sur le fichier F2 (AFC2)}

L'analyse AFC2 (fig 13) a été effectuée sur le fichier F2 obtenu en éliminant les 60 individus ayant exprimé plus de 5 "non choix» sur les 50 paires afin de tenter d'atténuer le biais expérimental mentionné plus haut.

Cette analyse n'a pas apporté de résultats vraiment spécifiques.

\section{Analyse sur le fichier F3 (AFC3)}

Ce fichier a été obtenu en éliminant de F2 les 123 individus ayant formulé au moins 1 «non choix». Aucun des 383 individus restants n'en a formulé.

La richesse du plan principal de l'analyse AFC3 nous permet de faire éclater le nuage des individus. Les diverses projections dans ce plan (fig 14) montrent que:

- les choix et les individus se répartissent sur l'ensemble du plan (fig 14a et 14b);

- les robots remplissent parfaitement leur rôle pour établir une grille d'interprétation. La figure $14 \mathrm{c}$ permet d'identifier 4 grands pôles.

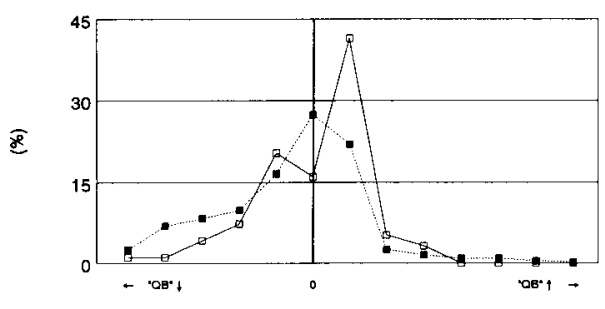

13 classes de répartition des effectifs suivant l'axe 1 de AFC1

Fig 11. AFC1 : répartition comparée des professionnels du bois et des consommateurs sur f'axe 1. $\square$ professionnels; consommateurs 


\section{0 individus \\ ayant exprimés \\ plus de \\ 5 "non choix"}

F1

Fichier complet 566 individus

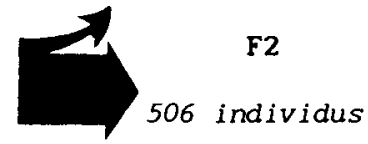

\begin{tabular}{|lll}
\hline \multicolumn{1}{|c}{ AFC2 } \\
A1 & $v p$ & 0,20 \\
& $c i$ & 11 \\
A2 & $v p$ & 0,07 \\
& $c i$ & 4 \\
& & \\
A3 & $v p$ & 0,07 \\
& $c i$ & 4 \\
$I T$ & 1,8 \\
IT &
\end{tabular}
123 individus

ayant exprimés

au moins

1 "non choix"

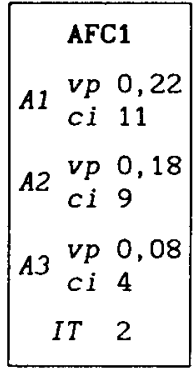
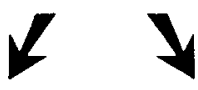
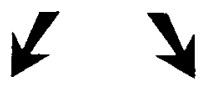

F2C

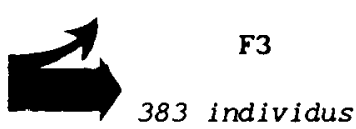

\begin{tabular}{|c|c|}
\hline & AFC3 \\
\hline$A 1$ & $\begin{array}{ll}v p & 0,20 \\
c i & 20\end{array}$ \\
\hline$A 2$ & $\begin{array}{ll}v p & 0,06 \\
c i & 6\end{array}$ \\
\hline A3 & $\begin{array}{ll}v p & 0,04 \\
c i & 4\end{array}$ \\
\hline & IT 1 \\
\hline
\end{tabular}

F1C

F1P

F2P

F3C

F3P

472 consom. 94 pro. 423 consom. 83 pro. 323 consom. 60 pro.

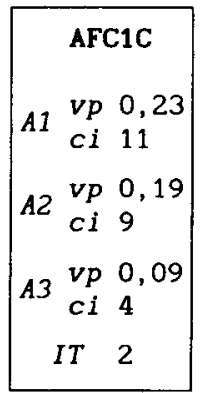

\begin{tabular}{|c|c|}
\hline & AFC1P \\
\hline & $\begin{array}{ll}v p & 0,22 \\
c i & 13\end{array}$ \\
\hline$A 2$ & $\begin{array}{ll}v p & 0,12 \\
c i & 7\end{array}$ \\
\hline$A 3$ & $\begin{array}{ll}\text { vp } & 0,10 \\
\text { ci } & 6\end{array}$ \\
\hline & $T \quad 1,7$ \\
\hline
\end{tabular}

\begin{tabular}{|lll} 
AFC2C \\
$A 1$ & $v p$ & 0,20 \\
& $c i$ & 12 \\
A2 & $v p$ & 0,07 \\
& $c i$ & 4 \\
A3 & $v p$ & 0,06 \\
& $c i$ & 4 \\
$I T$ & 1,7 \\
IT & & 1,7 \\
\end{tabular}

\begin{tabular}{|c|c|}
\hline & AFC2P \\
\hline & $\begin{array}{ll}v p & 0,20 \\
c i & 13\end{array}$ \\
\hline$A 2$ & $\begin{array}{l}\text { vp } 0,11 \\
\text { ci } 7\end{array}$ \\
\hline$A 3$ & $\begin{array}{ll}v p & 0,09 \\
c i & 6\end{array}$ \\
\hline & IT $\quad 1,5$ \\
\hline
\end{tabular}
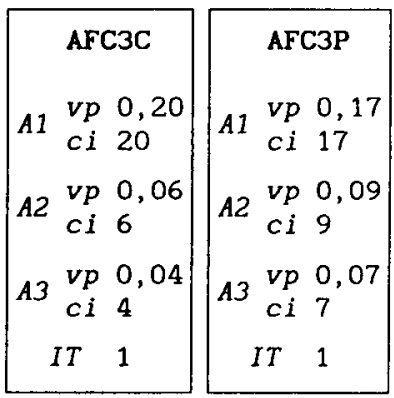

Fig 12. Les différentes analyses pratiquées. $A 1=$ axe $1 ; A 2=$ axe $2 ; A 3=$ axe $3 ; \mathrm{VP}=$ valeur propre des axes $; \mathrm{Cl}=$ contribution à l'inertie totale des axes exprimée en (\%).

Le pôle 1 correspond à la qualité la plus traditionnelle (nodosité faible, bon classement APECF, couleur homogène tirant sur le jaune). Le pôle 2 concerne les bois clairs orientés sur quartier et dont le figuré comprend beaucoup de cernes. Les pôles 3 et 4 sont les répliques inverses respectivement des pôles 1 et 2. 
A - AFC2: Nuage des Choix

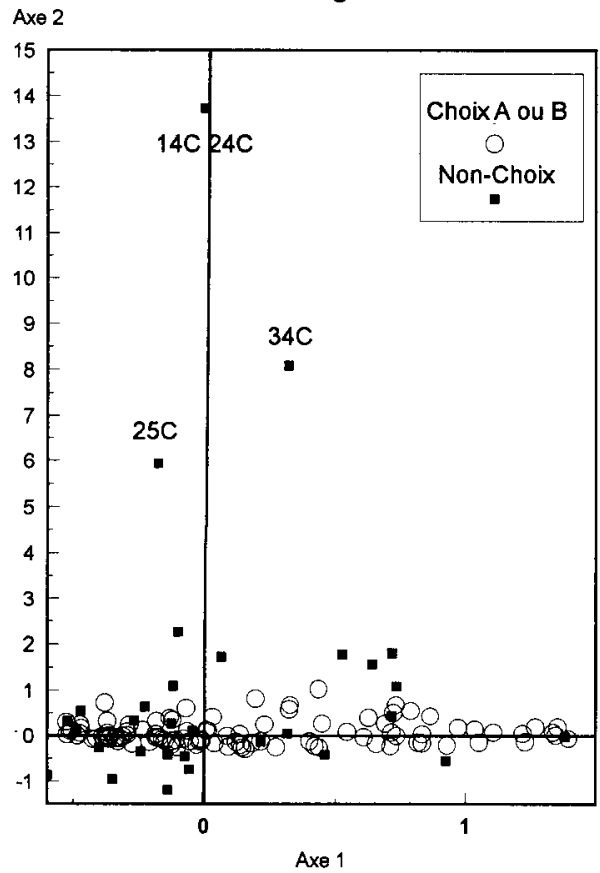

B - AFC2: Nuage des Individus

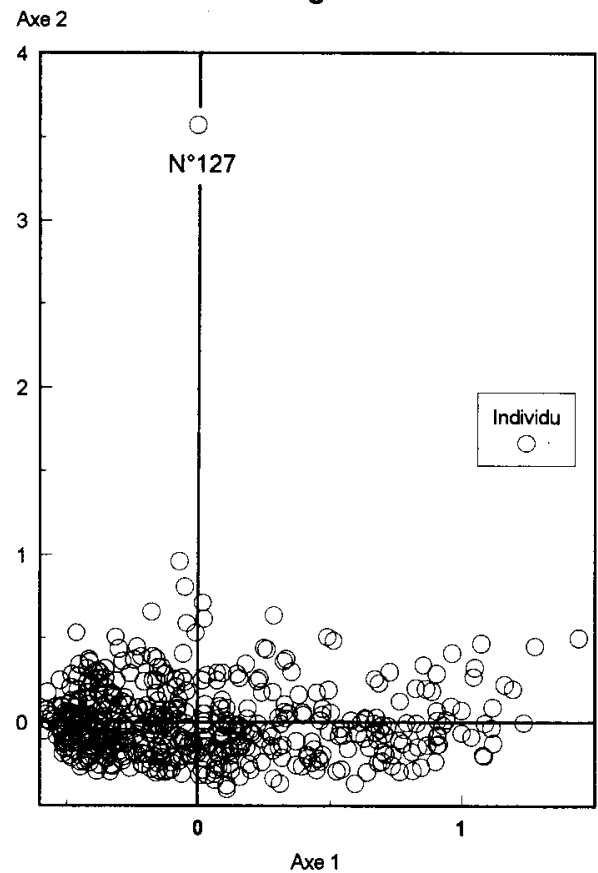

Fig 13. AFC2 : projections des choix et des individus dans le plan principal.

Cette grille permet de comprendre le moteur des choix formulés :

- les professionnels du bois, les bricoleurs et les hommes tendent plutôt vers le pôle 2 (fig 14d);

- il semble apparaître un gradient lié à l'âge des individus du pôle 3 (les plus jeunes) au pôle 1 (les plus âgés) ;

- en admettant que les 4 secteurs délimités par les axes 1 et 2 rassemblent les personnes tendant vers chacun des 4 pôles, le dénombrement séparé des professionnels du bois et des consommateurs indique des répartitions différentes (tableau II). Les goûts des consommateurs sont plus diffus que ceux des professionnels aux choix plus arrêtés. Par conséquent, même s'ils ne sont pas majoritaires, les consommateurs appréciant des bois "non traditionnels» constituent un marché non négligeable.
Bien qu'expliquant seulement $4 \%$ de l'inertie totale, l'axe 3 de l'AFC3 peut être interprété à l'aide des robots $\mathrm{DEN}^{-}, \mathrm{NBC}^{-}$, $\mathrm{LGC}^{-}$, et $\mathrm{h}^{*+}$ (bois dense à cernes larges et tirant vers le rouge) qui se retrouvent dans la partie positive de cet axe. Cela permet d'ébaucher une hiérarchisation des critères de qualité participant au choix des gens.

\section{Analyses sur les sous-fichiers "professionnels du bois» et «consommateurs»}

Les analyses effectuées sur les sousfichiers répertoriés sur la figure 12 ne font que conforter ces tendances. Les 3 fichiers professionnels (F1P, F2P, F3P) pèchent par une représentation des professionnels du bois de chêne peut-être pas assez 
A - AFC3: Nuage des Choix

Axe 2

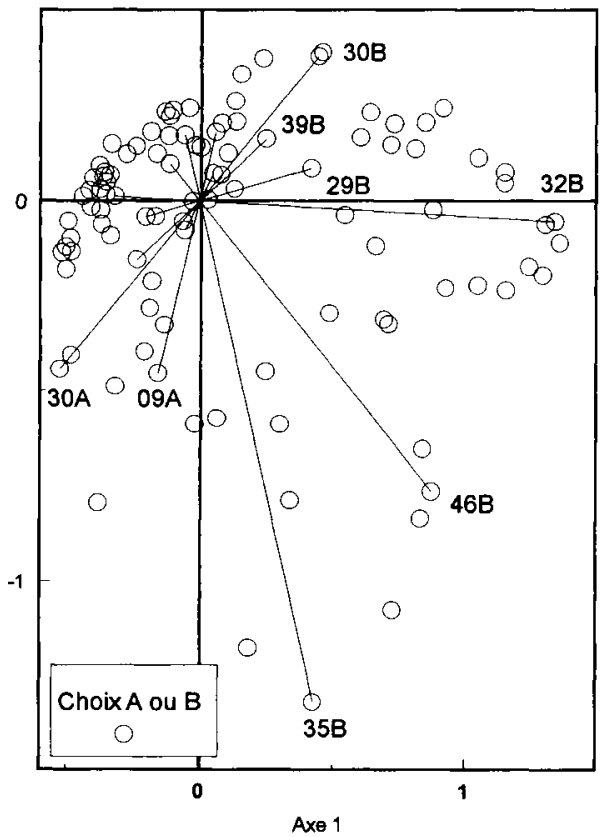

C - AFC3: Nuage des Robots

Axe 2

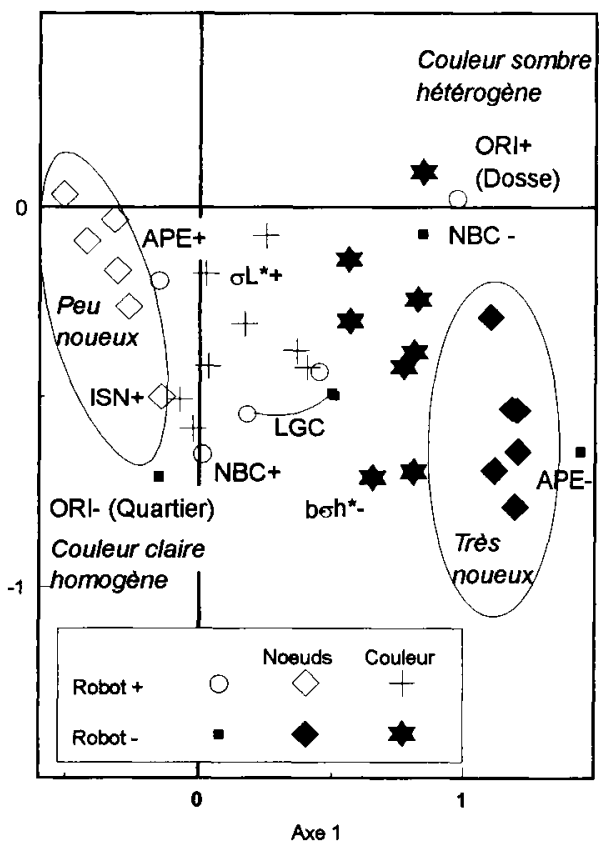

B - AFC3: Nuage des Individus

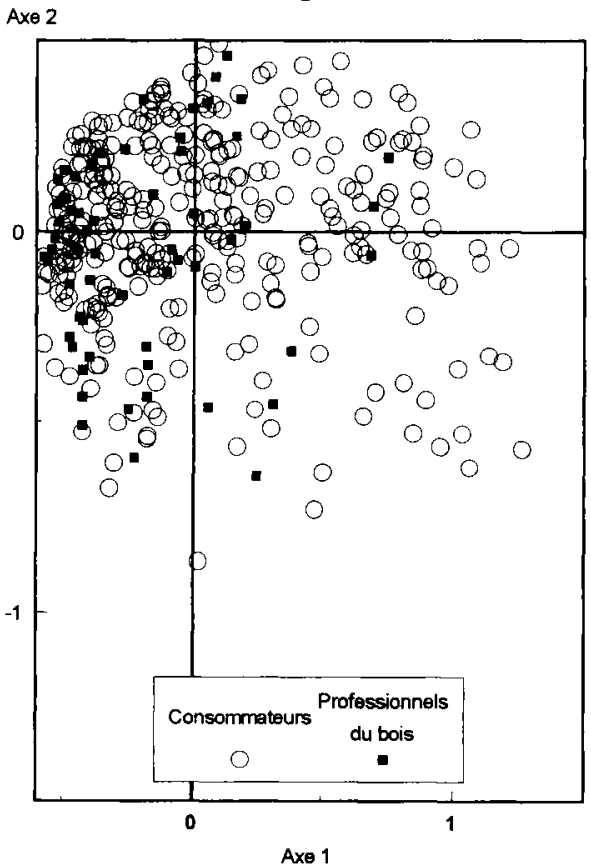

D - AFC3: Nuage des Profils Sociaux Axe 2

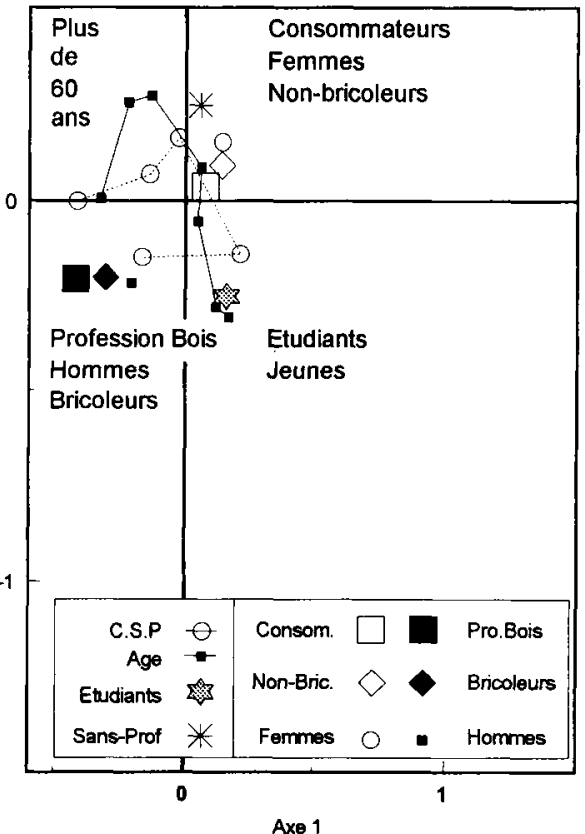

Fig 14. AFC3 : projections dans le plan principal. 
Tableau II. AFC3 : dénombrement séparé des consommateurs et des professionnels du bois dans les 4 secteurs du plan principal.

\section{Secteur 1}

- 19 professionnels $(32 \%)$

- 93 consommateurs $(28 \%)$

Secteur 2

- 25 professionnels (42\%)

- 87 consommateurs $(27 \%)$
Secteur 4

- 8 professionnels ( $13 \%)$

- 84 consommateurs $(26 \%)$

Secteur 3

- 7 professionnels (12\%)

- 60 consommateurs (19\%) ciblée, du fait de l'objectif initial de l'étude (fig 15). Cela revient à une population de consommateurs très avertis. Les grilles d'interprétation des AFC qui en découlent ne différent effectivement pas sensiblement des précédentes.

Les grandes tendances étant dégagées, des observations directes de paires particulièrement typées nous permettront d'affiner la hiérarchisation des critères de choix.

\section{Les enseignements obtenus par observations directes}

En s'appuyant sur les figures 16 et 17,3 classes de paires de frises ont été sélectionnées pour la nature des réponses formulées lors des sondages.

À l'intérieur de chacune de ces catégories illustratives, nous ne prenons en compte que les paires les plus marquées pour la

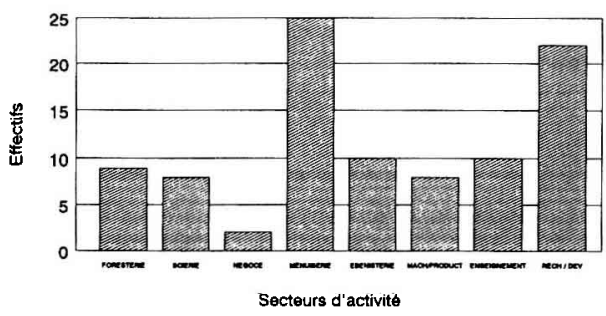

Fig 15. Répartition approximative des professionnels du bois interrogés par secteur d'activité. $\square$ professionnels ; consommateurs. fréquence des réponses (fichier complet) (tableau III). Ces catégories sont :

- première catégorie $\mathrm{C1}$ : appréciations nettes faisant la quasi-unanimité : la très grande majorité des personnes s'est prononcée en faveur d'une seule et même frise de chaque paire ; si tout le monde est d'accord, c'est qu'une frise de chaque paire est soit incontestablement belle, soit incontestablement laide ou renferme un vice grave ; cela doit nous permettre d'identifier les critères définitivement pénalisants, ou au contraire valorisants, pour le bois de chêne ; - seconde catégorie C2 : appréciations partagées : les choix se sont portés en nombre sensiblement égal sur l'une ou l'autre frise de chaque paire; nous sommes probablement en présence de types de frises pouvant contenter d'assez larges publics ;

- troisième catégorie C3 : nombreux «non choix" : un nombre important de personnes ne discerne pas de différence fondamentale entre les 2 frises de chaque paire; cela doit nous permettre d'identifier des critères fins ou cachés jouant sur le choix des personnes qui se sont déterminées malgré la grande ressemblance des 2 frises.

L'observation des paires de la catégorie C1 laisse apparaître que les frises comportant des nœuds altérés (creux, ouverts, éclatés, pourris) sont rejetées, quel que soit le cas de figure (35-46-27-10-43); il en va de même pour les frises sur quartier sur lesquelles les nœuds sont coupés dans le sens 


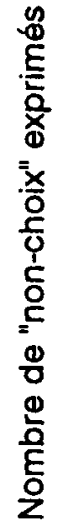

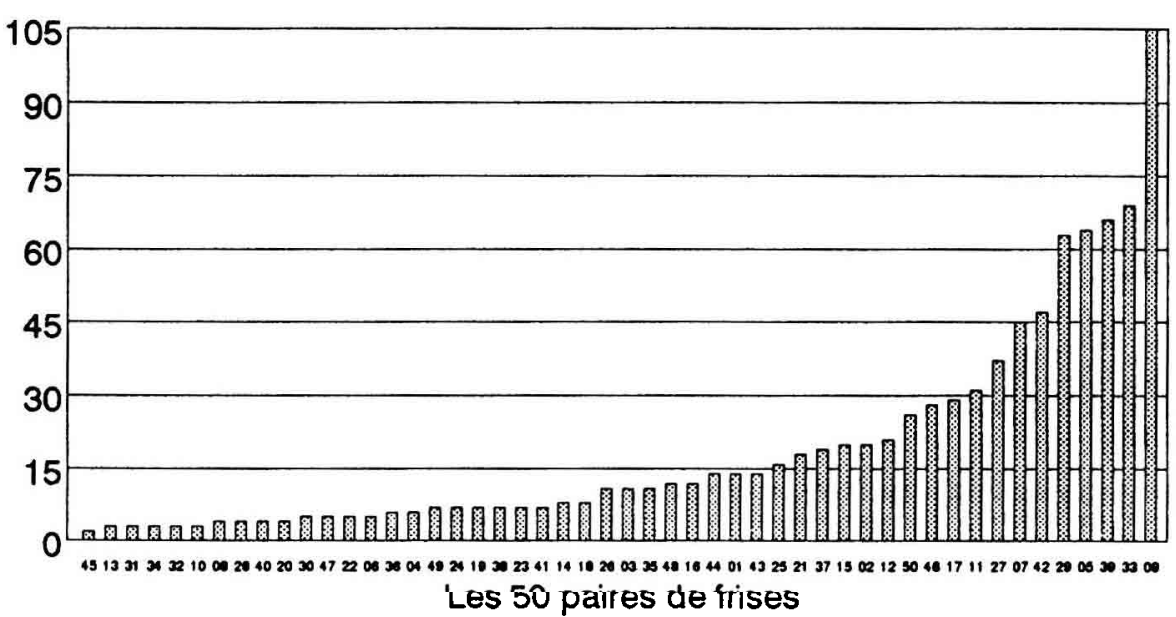

Fig 16. Fréquence des "non choix" exprimés sur chaque paire par l'ensemble des 566 personnes sondées.

0
$x$
0
$\frac{1}{0}$
0
0
0
0
$\frac{0}{2}$
1
$\frac{x}{0}$
$\frac{1}{0}$
0
0
0
0
$\frac{0}{2}$

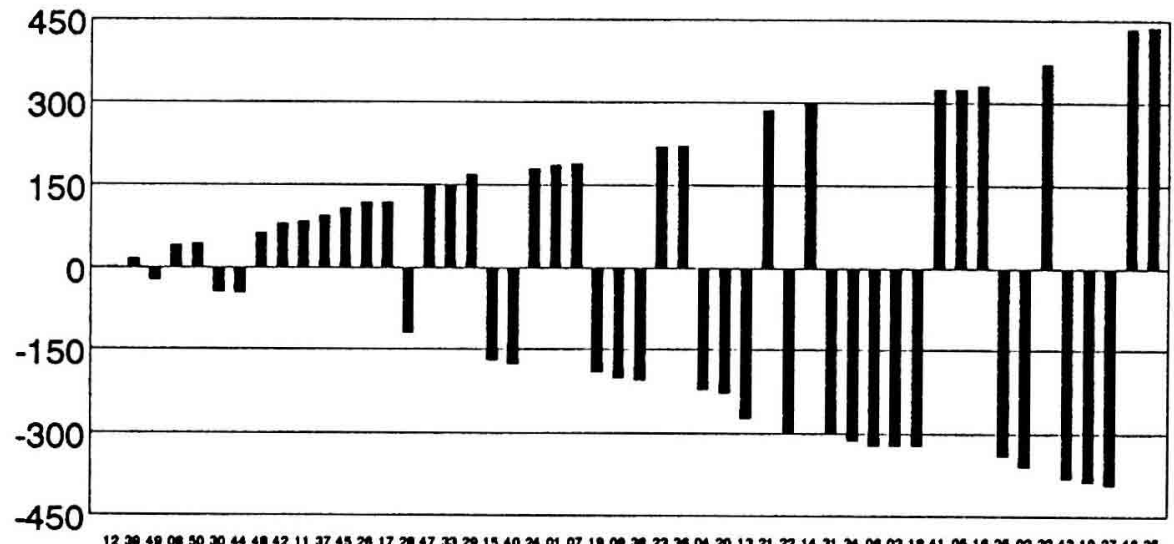

\section{Les 50 paires de frises}

Fig 17. Différence du nombre de choix A-B par paires pour l'ensemble des 566 personnes sondées.

du fil (nœuds plats) (35) ; les frises comportant des ncuds moyens à gros sont automatiquement rejetées face à une frise «QF1»(32).

Les avis sont donc tranchés quand une frise entachée par la présence de nœuds altérés ou noirs se retrouve face à une frise d'évidence plus harmonieuse.
Le type de nodosité intervient dans ces paires comme premier critère de choix. L'orientation de coupe n'est alors pas prise en compte.

L'observation des paires de la catégorie C2 laisse apparaître que :

- à surface de nœud équivalente, les comportements sont tout à fait partagés entre 
Tableau III. Caractéristiques des paires de frises ayant conduit à des réponses particulièrement typées.

\begin{tabular}{|c|c|c|c|c|c|}
\hline \multicolumn{2}{|c|}{$\mathrm{Cl}$} & \multicolumn{2}{|c|}{$\mathrm{C} 2$} & \multicolumn{2}{|c|}{$\mathrm{C3}$} \\
\hline Paires & $|A-B|$ & Paires & $|A-B|$ & Paires & $?$ \\
\hline 35 & 437 & 12 & 01 & & \\
\hline 46 & 434 & 39 & 16 & 09 & 105 \\
\hline 27 & 393 & 49 & 23 & 33 & 69 \\
\hline 10 & 387 & 08 & 42 & 39 & 66 \\
\hline 43 & 380 & 50 & 44 & 05 & 64 \\
\hline 32 & 369 & 30 & 45 & 29 & 63 \\
\hline 2 & 360 & 44 & 46 & & \\
\hline
\end{tabular}

$|A-B|=$ différence entre le nombre de choix exprimés sur la frise $A$ et la frise $B$ d'une paire donnée (valeur absolue). ? = nombre de «non choix».

un bois comportant beaucoup de petits nœuds en «pattes de chat» (bois ronceux) et un bois concentrant cette nodosité en 3 à 4 nœuds plus gros (12);

- si les différences sont minimes pour la nodosité (surface et types de nœuds équivalents sur les deux frises), l'orientation de coupe prime et la dosse est préférée (49, 30) ;

- les avis sont très partagés quand une frise sans noud fait face à une frise avec petits nœuds (30).

Deux "écoles" déterminées et fournies de consommateurs se dessinent: ceux préférant les figurés comportant des petits nœuds discrets (en «pattes de chat», voire un peu plus gros mais sains et peu nombreux) et ceux excluant tout nœud.

Enfin, l'observation des paires de la catégorie C3 laisse apparaître que les frises constituant chacune des 5 paires de $\mathrm{C} 3$ présentent 2 à 2 la même orientation, sensiblement les mêmes types de nœuds répartis à la même fréquence suivant le même schéma ; une frise «l'emporte» nettement sur une autre présentant des variations importantes de teinte (33-29) ; sur une paire jumelle QF1, le grain fin est préféré au grain plus large (39).

La largeur des cernes n'est prise en compte par l'observateur que lorsqu'elle reste le seul facteur de variabilité.

Une hétérogénéité de teinte est toujours sanctionnée (fig 18).

\section{DISCUSSION}

Les AFCM ont permis de constater:

- l'absence de monolithisme dans les réponses (dispersion des "points individus" le long de l'axe qualité) : tous les goûts existent et aucun groupe ne prédomine très largement ;

- l'importance primordiale donnée aux nœuds et à l'orientation de coupe dans le jugement des personnes ; les comportements sont partagés face à ces 2 critères ; le classement APECF discrimine parfaitement les 2 populations ;

- les grandes tendances dégagées ci-dessus sont légèrement accentuées lorsqu'on ne considère que la sous-population des professionnels (fig 14); 

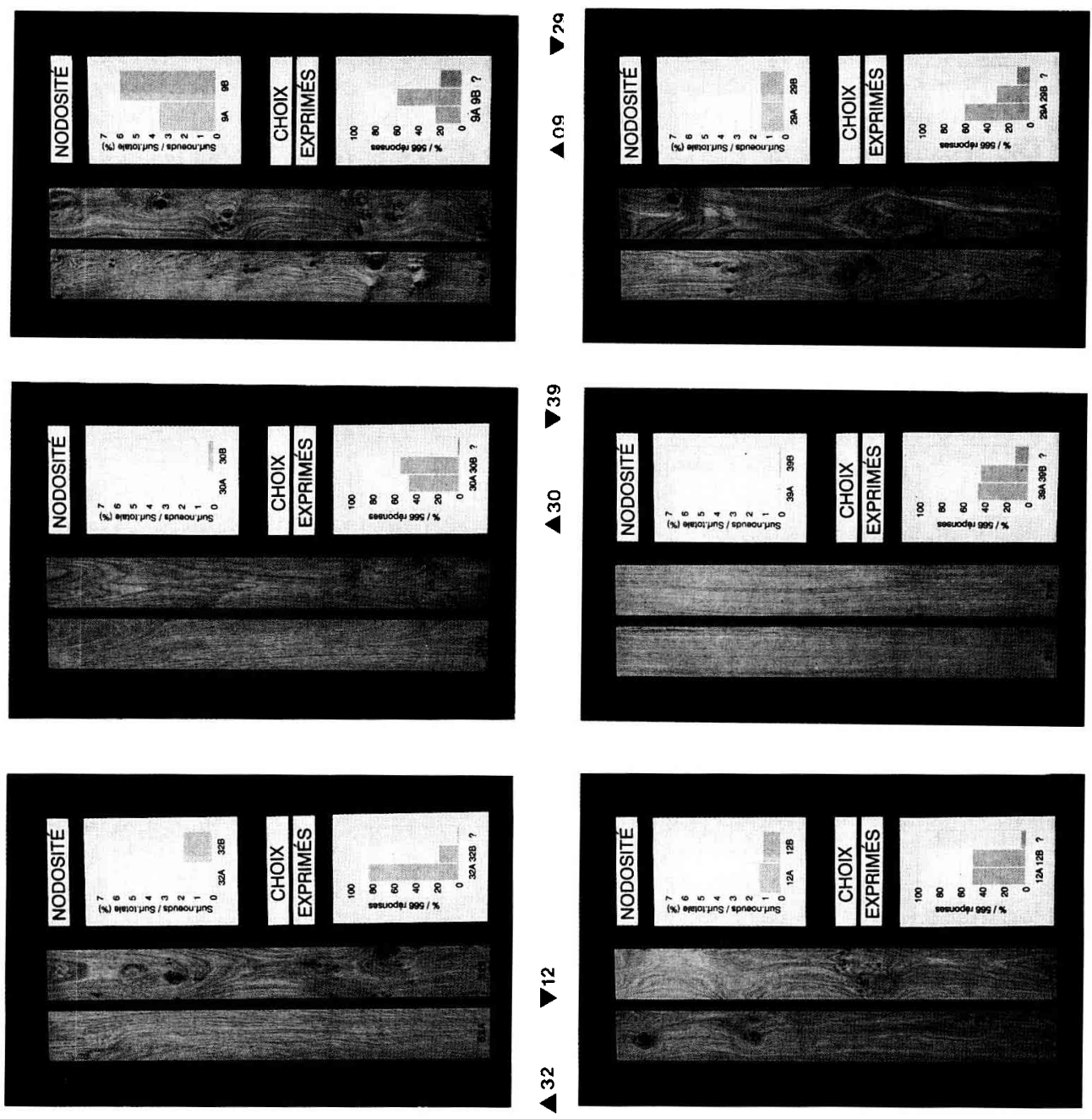

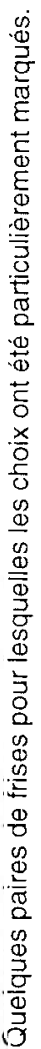
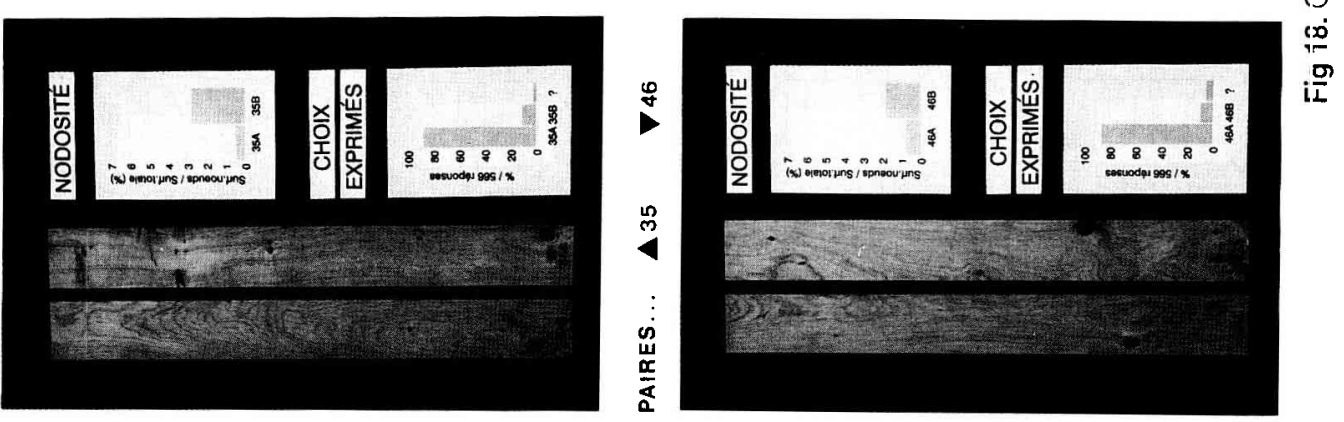
- que la couleur intervient à 2 niveaux : la clarté métrique et les écarts types des coordonnées chromatiques; ainsi, les bois de couleur claire et homogène - majoritairement sur quartier et à grain fin - et ceux de couleur sombre et hétérogène sont appréciés par des publics différents ;

- que le critère «densité du bois» et les coordonnées chromatiques $a^{*}$ et $b^{\star} n^{\prime}$ apparaissent pas jouer un rôle de premier plan dans le choix ;

- que les femmes tendent à préférer des bois plus noueux - en nombre de nœuds et en surface-que les hommes ;

- que les personnes habituées à bricoler et les professionnels du bois tendent à rejeter systématiquement les nœuds ;

- un comportement différent suivant les classes d'âge: plus la population vieillit, plus les choix sont faits suivant les critères traditionnels de qualité ; ce comportement assez graduel s'observe aussi avec les professionnels du bois ;

- que les catégories socio-professionnelles ne se différencient pas significativement par leur comportement.

Les observations directes ont permis : i) d'établir une hiérarchie dans les critères participant aux choix formulés par les personnes. Les diverses observations convergent pour valider l'ordre de préséance suivant:

- nodosité >

- orientation de coupe >

- clarté et homogénéité de teinte >

- largeur des cernes ;

ii) d'identifier 2 grandes populations de consommateurs : ceux rejetant toute trace de nœud dans un bois de chêne et ceux préférant le chêne faiblement noueux - de type QF1bis, voire QF2 dans certaines configurations (nœuds parfaitement sains, ...) à des bois exempts de tout nœud; cette population, plutôt jeune, semble être majoritairement composée de femmes ; iii) de mettre en évidence 2 types de réaction face au premier choix (QF1) : QF1 est préféré face à des bois trop noueux de type QF2, QF3 quelle que soit l'orientation de coupe ; les avis sont partagés face à une frise QF1bis (tolérance de 2 petits nœuds par mètre linéaire) qui l'emporte quand elle est sur dosse dominante.

Les cuisinistes, ébénistes et menuisiers sont assurés de trouver une large clientèle en intégrant des QF1bis, voire des QF2, en parement dans leurs réalisations.

\section{Perspectives}

Tous nos résultats sont conformes aux tendances pressenties, ainsi qu'au bon sens. L'intérêt de cette étude est d'avoir apporté des preuves objectives sur les critères guidant les choix des consommateurs français.

Mazet (1988) a montré que le comportement des professionnels du bois dépend de leur nationalité (France/Italie). II en va probablement de même pour les consommateurs. Le prolongement de la présente enquête, envisagé par G Janin en Allemagne, permettra de le vérifier.

Le protocole d'enquête utilisé est perfectible : demander aux personnes de juger des pièces de bois dans l'absolu, en s'eftorçant de s'affranchir de tout type d'emploi éventuel (parquet, huisserie, meuble...) peut paraître foncièrement irréaliste. Cela a pourtant permis de sérier les problèmes en autorisant l'établissement d'une hiérarchie dans les critères de choix.

L'expérience pourrait être renouvelée, sur un échantillonnage réduit, en proposant aux personnes de passer plusieurs fois : à chaque passage, un emploi bien précis de ces bois leur serait proposé en perspective. Les réponses seraient probablement différentes. En effet, certains figurés peuvent être jugés à la fois "fatigants" en ébénisterie et "chaleureux» en parqueterie. 
Un jugement sur des frises ne permet pas forcément de se faire une bonne idée de l'aspect du bois mis en œuvre. Pourquoi alors ne pas soumettre au jugement quelques répliques des mêmes produits finis fabriqués avec des bois de qualités différentes?

Enfin, d'autres relevés objectifs (comme le type des nœuds ou la surface des rayons ligneux) permettraient d'affiner l'identification des critères de choix des personnes.

\section{REMERCIEMENTS}

Nous tenons à remercier Jean-Claude Butaud (ENSAM), Claude Houssement (INRA), Gérard Janin (INRA), et Dominique Juillot (Scieries réunies du Châlonnais) pour leurs conseils et aides matériels ; Étienne Claude, Yvette Claude, Anne Mailland, Jeanne Messeleka, et les étudiants de la formation "bois de I'ENSAM" pour avoir pris en charge un grand nombre de sondages ; ainsi que toutes les personnes ayant pris part au sondage.

\section{RÉFÉRENCES}

APECF (1989) Guide pratique d'emploi des sciages de chêne et de hêtre français. AFME, FNB, CTBA, DERF, $98 p$

Brun-Chaize MC (1978) Le paysage forestier, analyse des critères de préférence du public à partir de photographies. Les Cahiers de I'Analyse des Données, vol III, $\pi^{\circ} 1,65-78$

Dervin C (1990) Comment interpréter les résultats d'une analyse factorielle des correspondances ? Institut Technique des Céréales et des Fourrages (ITCF), $75 \mathrm{p}$

Janin $G$ (1987) Mesure de la couleur du bois. Intérêt forestier et industriel. Ann Sci For 44 (4), 455-472

Kowaliski $P$ (1990) Vision et mesure de la couleur. Masson, Paris, $255 p$

Marchal R, Mothe F (1992) Appréciation des différentes classes du bois de chêne par le consommateur trançais. Résultats intermédiaires. Communication à la Troisième journée professionnelle filière bois de l'ENSAM, 12 février 1992, Cluny, $20 p$

Mazet JF (1988) Couleur et qualité des placages de chêne et étude de leur comportement photochimique. Thèse de Doctorat de l'université Nancy 1, spécialité sciences du bois, $136 \mathrm{p}$

Mazet JF (1989) Recherche de critères pour l'aspect (dessin et couleur) des placages de bois de chêne. Les cahiers de l'Analyse des Données 14 (3), 365-376

Mazet JF, Janin G (1990) La qualité de l'aspect des placages de chênes : mesure de couleur et critères d'appréciation des professionnels français et italiens. Ann Sci For 47 (3), 255-268

STAT-ITCF (1991) Version 5 du logiciel de statistique de I'ITCF. Institut technique des céréales el des fourrages (ITCF), Paris 